\title{
Pattern of Rheumatic Disorders Among Sudanese Patients-Khartoum State
}

\author{
Hyder Mirghani ${ }^{2,}$, Ahmad Suleiman ${ }^{1}$ \\ ${ }^{1}$ Department of Medicine, Faculty of Medicine, University of Tabuk, Tabuk, Kingdom of Saudi Arabia \\ ${ }^{2}$ Department of Medicine, Faculty of Medicine, Northern Border University, Arar, Kingdom of Saudi Arabia \\ Email address: \\ shyder63@hotmailcom (H. Mirghani),shyder63@hotmailcom (A. Suleiman) \\ ${ }^{*}$ Corresponding author
}

\section{To cite this article:}

Hyder Mirghani, Ahmad Suleiman. Pattern of Rheumatic Disorders Among Sudanese Patients-Khartoum State. International Journal of Neurologic Physical Therapy. Vol. 3, No. 3, 2017, pp. 17-20. doi: 10.11648/j.ijnpt.20170303.11

Received: April 18, 2017; Accepted: May 10, 2017; Published: May 22, 2017

\begin{abstract}
The current study aimed to assess the pattern of rheumatic disorders in Omdurman Teaching Hospital, Khartoum, Sudan. This cross-sectional descriptive study was conducted among Sudanese patients who attended the outpatient rheumatic clinic in Omdurman Teaching Hospital in Sudan during the period from March to September 2013. A structured questionnaire was designed to provide a profile of Sudanese rheumatic patients; the information collected were age, sex, occupation, marital status if receiving follow-up, social status, disabilities and associated chronic diseases. The participants signed a written informed consent, and the Statistical Package for Social Sciences was used for data analysis. Ethical clearance was obtained from the ethical committee of Omdurman Teaching Hospital. The study included a total of 248 subjects; male to female ratio

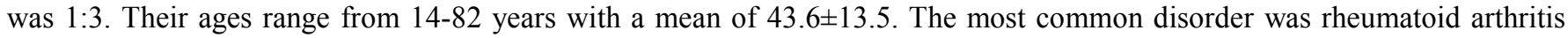
$(60.5 \%)$, followed by systemic lupus erythematosus $(12.9 \%)$, and gouty arthritis (8.9\%). Rheumatoid arthritis was most prevalent in female $76.6 \%$ versus $23.4 \%$, SLE was found in $87.5 \%$ of females as compared to male $12.5 \%$, while gouty arthritis was more common in males $81.8 \%$ versus $18.2 \%$. Concerning disability, $36.3 \%$ of patients were disabled, $25 \%$ of them had left work, and $11.3 \%$ had left school. Twenty percent of patients had hypertension, $15.3 \%$ had diabetes mellitus, $10 \%$ had bronchial asthma, and 5.6\% had thyroid disorders. The commonest rheumatic disease was rheumatoid arthritis, followed by SLE and gouty arthritis. Further larger studies are needed to characterize the pattern of rheumatic disorders in this country.
\end{abstract}

Keywords: Pattern, Rheumatic Disorders, Sudan

\section{Introduction}

Rheumatic diseases in Sudan are old, in support of this view; is a case of erosive arthritis reported in a skeleton from Kulubnarti, Republic of the Sudan (c. 700-1450 A. D) [1], but data concerning the pattern of these disorders are scarce in Sudan.

Rheumatic diseases are a spectrum of disorders that predominantly affect joints, skin, blood cells, kidneys, lungs and the central nervous system. Rheumatic diseases have a tremendous impact on both individuals and society in from of decreased quality of life, lost productivity, and increase the cost of health care; this can be expected to rise as the population ages. One of the challenges of studying osteoarthritis and rheumatoid arthritis and rheumatic diseases, in general, is driving epidemiological data that is useful for better understanding of factors that contribute to the initiation and progression of this diseases [2].

Rheumatic diseases are prevalent worldwide causing a lot of morbidity and mortality. Musculoskeletal disorders are among the principal causes of physical disability and expend a large amount of health resources globally [3] Musculoskeletal disorders are the second cause of healthy years lost to morbidity and adversely impact the quality of life [4]. Early accurate diagnosis is vital to avoid inefficient use of resources, such as additional testing and unnecessary referrals.

Rheumatic diseases are among the oldest diseases recognized. The classification of rheumatic diseases is sometimes difficult due to unknown etiology and heterogeneity in their clinical presentation. Osteoarthritis and rheumatoid arthritis are the two most common rheumatic diseases accounting for a significant percentage of disability 
worldwide. Studies addressing the epidemiology and factors that contribute to the initiation and progression of these diseases are of paramount importance for the appropriate approaches to patient's management and control of these diseases, and to reduce the patient's suffering and the burden on patients and the whole society [2].

Rheumatic disorders are common diseases worldwide that result in significant mortality and morbidity. In the United States, osteoarthritis affects 27 million, eight are affected by gout arthritis, 1.3 million had rheumatoid arthritis, and up to 322 thousands had systemic lupus erythematosus. The prevalence of doctor diagnosed arthritis is $21 \%$ [5].

The highest prevalence of rheumatic disorders is in the South America and the Caribbean countries where 23.8-56\% of the populations are affected, the prevalence is $15-24 \%$ in Australia and New Zealand, while up to $13 \%$ of the United Kingdom are affected [6].

The prevalence of rheumatic disorders is widely unknown in Sudan, but it is observed that a lot of outpatients visits is due to rheumatic complaints. Thus we conducted this research to study the pattern of rheumatic diseases in Omdurman Teaching Hospital, Sudan.

\section{Subjects \& Methods}

This cross-sectional descriptive study carried out in a rheumatic outpatients clinic in Omdurman Teaching Hospital in Sudan.

Omdurman Teaching Hospital is a Central Hospital serving a large area in Sudan extending from Khartoum to the far west. receiving referrals from Primary Health Care Centers and General Hospitals. The study was conducted during the period from March to September 2013. The patients were approached in a ratio of $1 ; 1$, the number stands at 248 patients during the stated period. All the participants signed a written informed consent.

The patients were already diagnosed and came for routine follow-up, a questionnaire was designed to provide a profile of Sudanese rheumatic patients. The information collected, involved age, sex, occupation, marital status if receiving follow-up, social status, disabilities, associated chronic diseases and extra-articular manifestations of rheumatoid arthritis that include eye manifestations, serositis, lung fibrosis and neurological manifestations.

A full explanation of the purposes of, nature and procedures of the study were first conveyed to them. The potential participants were explicitly assured that their participation is entirely voluntary, any data obtained would be treated confidentially and only for the purpose of the research. The Statistical Package for Social Sciences (SPSS) was used for data analysis; the data were presented as mean \pm SD and percentages. The ethical committee of Omdurman Teaching Hospital approved the research.

\section{Results}

The study included 248 patients, 190 of them were women
(76.6\%); their ages range was $14-82$ with a mean of 43.6 . The majority of patients were married $(74.6 \%)$. In the present study, more than two-thirds $(67.3 \%)$ were housewives, $18.1 \%$ were laborers, $8.5 \%$ were employees, while students constituted $6.1 \%$. The demographic characteristics of the patients were shown in table1. Physical disability affected ninety patients $(36.3 \%)$ of the study group, of them, $62(25 \%)$ left work and $28(11.3 \%)$ Left school, Table 2. Regarding other co-morbidities, fifty-two (20.1\%) were hypertensive, $38(15.3 \%)$ were diabetic; other associated chronic diseases were depicted in Table 3. The majority of patients 150 $(60.5 \%)$ had rheumatoid arthritis, followed by SLE (12.9\%), and gouty arthritis (8.9\%). In the current study rheumatoid arthritis and SLE were commoner among females (46.3\% vs. $14.2 \%$ and $11.3 \%$ vs. $1.2 \%$ respectively), while gouty arthritis was more among males $(7.2 \%$ vs. $1.7 \%)$. The distribution of other rheumatic diseases is shown in Table (4).

Table 1. Patients' characteristics in the study group.

\begin{tabular}{ll}
\hline Characteristic & N (\%) \\
\hline Gender & $58(23.4 \%)$ \\
Male & $190(76.6 \%)$ \\
Female & \\
Age & $14-82$ \\
Range & $43.6 \pm 13.5$ \\
Mean & \\
Occupation & $167(67.3 \%)$ \\
Housewife & $45(18.1 \%)$ \\
Laborer & $21(8.5 \%)$ \\
Employee & $15(6.1 \%)$ \\
Student & \\
Social class & $53(21.4 \%)$ \\
Moderate & $195(78.6 \%)$ \\
Low & \\
Follow-up & $202(81.4 \%)$ \\
Yes & $465(18.6 \%)$ \\
No & \\
Marital status & \\
Married & \\
Unmarried & \\
\hline & \\
\hline
\end{tabular}

Table 2. Disabilities among the study group.

\begin{tabular}{ll}
\hline disability & No $(\%)$ \\
\hline Left work & $62(25 \%)$ \\
Left school & $28(11.3 \%)$ \\
Total & $90(36.3 \%)$ \\
\hline
\end{tabular}

Table 3. Associated diseases among the study group.

\begin{tabular}{ll}
\hline Disease & $\mathbf{N}(\mathbf{\%})$ \\
\hline Hypertension & $52(20.1 \%)$ \\
Diabetes & $38(15.3 \%)$ \\
Thyroid disease & $14(5.6 \%)$ \\
Bronchial asthma & $10(4 \%)$ \\
\hline
\end{tabular}


Table 4. Distribution of rheumatic diseases among the study group.

\begin{tabular}{llll}
\hline Disease & $\begin{array}{l}\text { Male N } \\
(\%)\end{array}$ & $\begin{array}{l}\text { Female } \\
\mathbf{N}(\%)\end{array}$ & $\begin{array}{l}\text { Total N } \\
(\%)\end{array}$ \\
\hline Rheumatoid arthritis & $35(14.2)$ & $115(46.3)$ & $150(60.5)$ \\
SLE & $4(1.6)$ & $28(11.3)$ & $32(12.9)$ \\
Gouty arthritis & $18(7.2)$ & $4(1.7)$ & $22(8.9)$ \\
Undiagnosed & $4(1.7)$ & $8(3.1)$ & $12(4.8)$ \\
Osteoarthritis & $2(0.8)$ & $6(2.5)$ & $8(3.3)$ \\
Spondyloarthropathy & $3(1.2)$ & $2(0.8)$ & $5(2)$ \\
Juvenile chronic arthritis & $2(0.8)$ & $3(1.2)$ & $5(2)$ \\
Behcet's disease & $2(0.8)$ & $3(1.2)$ & $5(2)$ \\
Scleroderma & $0(0.0)$ & $2(0.8)$ & $2(0.8)$ \\
Myositis \& Antiphospholipid & $5(2)$ & $2(0.8)$ & $7(2.8)$ \\
syndrome & &
\end{tabular}

\section{Discussion}

The different demographic characteristics of patients pose a high impact on their health status. In fact, the old age itself adds a lot in term of disabilities to patients with rheumatic disorders.

In the present study the most common rheumatic disease was rheumatoid arthritis $60.5 \%$, followed by systemic lupus erythematosus $12.5 \%$, gouty arthritis $8.9 \%$ and osteoarthritis $3.3 \%$ of patients, similarly $\mathrm{Ng}$ et al. [7] showed a higher prevalence of rheumatoid arthritis and SLE in a study conducted in Singapore. In contrast to the current study, community-based studies [8] revealed that the osteoarthritis is the most common rheumatic disorder, this can be explained by the relatively young age group in the current study as well as the fact that most cases of osteoarthritis are treated by orthopedic surgeons or general physicians and not usually referred to rheumatic clinics.

In consistency with the current result is a study published by Vanhoof et al. [9] and showed that $49 \%$ of patients returning to the referred clinic had an inflammatory rheumatic disease, 33\% had osteoarthritis, and 19\% had metabolic arthritis. In the current study $76.6 \%$ of rheumatoid arthritis, 87.5 of SLE patients, $75 \%$ of osteoarthritis were females, while male constitutes the majority of gouty arthritis $(81.8 \%$,$) and in agreement with previous studies [9].$

The present study reported that $20.1 \%$ of patients were hypertensive, and $15.3 \%$ were affected by diabetes mellitus. This is important in term of increased cardiovascular mortality and morbidity. Furthermore patients with rheumatic diseases are at high risk of cardiovascular disease due to the increased inflammatory and proinflammatory substances, that enhance the formation and progression of atherosclerosis [10].

In the present study, physical disability was reported in $36.3 \%$ of patients, previous literature concluded the increased overall mortality, cardiovascular mortality, and increase atherosclerosis and cardiovascular events among patients affected by disability [11]. Given the above, patients with rheumatic diseases deserve more care in term of vascular risk assessment, prevention, and the introduction of the appropriate management when indicated.

Extra-articular manifestations are associated with increased risk of developing coronary artery disease as well as peripheral arterial disease, and is a predictor of both overall mortality and cardiovascular mortality, indicating that systemic inflammation is a major determinant of vascular comorbidity in rheumatoid arthritis, systemic lupus erythematosus and rheumatic diseases in general [12-14]. In the present study, nearly one-fifth of the patients had extraarticular manifestations. The limitations of the study were the relatively small size of the study group, and the fact that the study was conducted at a single outpatient rheumatic clinic, so generalization cannot be insured.

\section{Conclusion}

The most common rheumatic disorder was rheumatoid arthritis, followed by SLE and gouty arthritis. Women were more affected by rheumatoid arthritis and SLE, while gouty arthritis was commoner among males. The current study presented a sample of Sudanese rheumatic patients with unwanted features like disabilities and extra-articular manifestation putting them at a high mortality, further larger studies are needed to characterize the epidemiology of rheumatic disorders in this country.

\section{References}

[1] Kilgore L. Possible case of rheumatoid arthritis from Sudanese Nubia. Am J Phys Anthropol. 1989 Jun; 79 (2): 17783. P M I D: 2662779.

[2] Sangha O. Epidemiology of rheumatic diseases. Rheumatology 2000; 39(supply): 3-12.

[3] Bagher O M, Golbarg M, Hossein S. Pattern of rheumatic diseases in two outpatient clinics in Iran: similarities with some different features. ndian J Med Sci. 2011 Jan; 65 (1): 7 17. doi: 10.4103/0019-5359.103160.

[4] Lowe D B, Taylor M J, Hill SJ. Associations between multimorbidity and additional burden for working-age adults with specific forms of musculoskeletal conditions: a crosssectional study. B M C Musculoskelet Disord. 2017 Apr 4; 18(1): 135. doi: 10.1186/s12891-017-1496-2.

[5] Helmick C G, Felson D T, Lawrence R C, et al. Estimates of the prevalence of arthritis and other rheumatic conditions in the United States- Part I. Arthritis \& Rheum. 2008; 58 (1): 15-25.

[6] Charles G H, David T F, Reva C etal. Estimates of the prevalence of arthritis and other rheumatic conditions in the United States- Part I. Arthritis \& Rheum 2008; volume58 nol.

[7] $\mathrm{Ng} \mathrm{X,} \mathrm{Low} \mathrm{A} \mathrm{H,} \mathrm{Chew} \mathrm{L} \mathrm{C,} \mathrm{Chong} \mathrm{Y} \mathrm{Y,} \mathrm{Fong} \mathrm{K} \mathrm{Y,} \mathrm{Lui} \mathrm{N} \mathrm{L,}$ Sim E, Tan Y K, Yoong J, Thumboo J. Disease patterns of rheumatology outpatients seen in a tertiary hospital serving a multi-ethnic, urban Asian population in Singapore. Int J Rheum Dis. 2013 Jun; 16(3): 273-8. doi: 10.1111/1756-185x. 12016. Epub 2012 Dec 17.

[8] Rose W, AileenM, Elizabeth B etal. Prevalence diseases the world growing burden and implications for health care needs Rheumatoid arthritis. Clin Evid Concise 2003; 10: 274-6.

[9] Vanhoof J, Declerck K, Geusens P. Prevalence of rheumatic diseases in a rheumatological outpatient practice. Ann Rheuma Dis 2002; 61: 453-455. 
[10] Solomon D H, Curhan G C, Rimm E B et al. Cardiovascular risk factors in women with and without rheumatoid arthritis. Arthritis Rheum. 2004; 50: 3444-9.

[11] Young A, Koduri G, Batley $\mathrm{M}$ et al. Mortality in rheumatoid arthritis. Increased in the early course of the disease, in ischaemic heart disease and pulmonary fibrosis. Rheumatology (Oxford) 2007; 46: 350-59.

[12] Turesson C, Mc Clelland R L, Christianson T J et al. Severe extra-articular disease manifestations are with an increased risk of first ever cardiovascular events in patients with rheumatoid arthritis. Ann Rheum Dis. 2007; 66: 59-64.

[13] JLiang K P, Liang K V, Matteson E L et al. Incidence of noncardiac vascular disease in rheumatoid arthritis and relationship to extra-articular disease manifestations. Arthritis Rheum. 2006; 54: 642-8.

[14] Gabriel S E, Crowson C S, Kremers H M et al. Survival in rheumatoid arthritis: a population- based analysis of trends over 40 years. Arthritis Rheum. 2003; 48: 54-8. 